\title{
Alternating Copolymerization of Vinyl Acetate with Carbon Monoxide
}

\author{
Takuya Kochi, Akifumi Nakamura, Hirotsugu Ida, and Kyoko Nozaki* \\ Department of Chemistry and Biotechnology, Graduate School of Engineering, \\ The University of Tokyo, 7-3-1 Hongo, Bunkyo-Ku, Tokyo 113-8656, Japan, \\ and Tsukuba Research Laboratories, Kuraray Company, Ltd., \\ 41, Miyukigaoka, Tsukuba, Ibaraki 305-0841, Japan
}

\section{Experimental Section}

General Methods. All manipulations were carried out using the standard Schlenk technique under argon purified by passing through a hot column packed with BASF catalyst R3-11. ${ }^{1} \mathrm{H}(500 \mathrm{MHz}),{ }^{13} \mathrm{C}(126 \mathrm{MHz})$ and ${ }^{31} \mathrm{P}$ NMR $(202 \mathrm{MHz})$ spectra were recorded on a JEOL JNM-ECP-500 spectrometer. Elemental analysis was performed by the Microanalytical Laboratory, Department of Chemistry, Faculty of Science, the University of Tokyo. Size exclusion chromatography (SEC) analyses were carried out at $40{ }^{\circ} \mathrm{C}$ with a GL Sciences instrument (model PU 610 high-performance-liquid-chromatography pump, CO 631A liquid chromatography column oven, and RI 713 refractive-index detector) equipped with two columns (Shodex KF-804L) by eluting the columns with tetrahydrofuran (THF) at $1 \mathrm{~mL} / \mathrm{min}$. Matrix-assisted laser desorption/ionization time-of-flight mass spectrometry (MALDI-TOF MS) was performed on an Applied Biosystems BioSpectrometry Workstation model Voyager-DE STR spectrometer using 2-(4-hydroxyphenylazo)benzoic acid (HABA) as a matrix. Fast atom bombardment mass spectrometry (FAB-MS) was carried out on a JEOL JMS-700 spectrometer using PEG calibration and NBA matrix solvent. $\quad 2$-\{di(2-methoxyphenyl)phosphino\}benzenesulfonic $\quad$ acid $\quad(\mathbf{1 a})^{1}$ 2-(diphenylphosphino)benzenesulfonic acid (1b) ${ }^{2}$ and $\left[{ }^{i} \mathrm{Pr}_{2} \mathrm{EtNH}\right]\left[\left\{0-\left(\mathrm{Ph}_{2} \mathrm{P}\right) \mathrm{C}_{6} \mathrm{H}_{4} \mathrm{SO}_{3}\right\} \mathrm{PdMeCl}\right](\mathbf{3 b})^{3}$ were prepared according to literature procedures. 


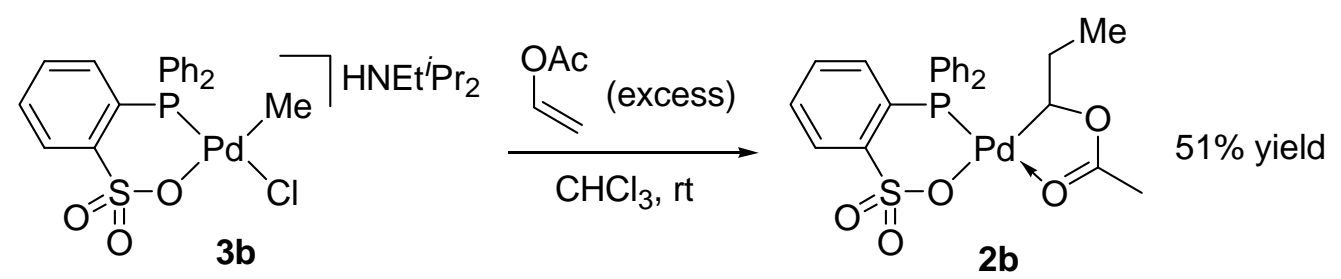

Preparation of $\left[\left\{o-\left(\mathrm{Ph}_{2} \mathrm{P}\right) \mathrm{C}_{6} \mathrm{H}_{4} \mathrm{SO}_{3}\right\} \mathrm{Pd}\left\{\mathrm{CH}(\mathrm{OAc}) \mathrm{CH}_{2} \mathrm{CH}_{3}\right\}\right]$ (2b). To a mixture of $181 \mathrm{mg}$ of $\mathbf{3 b}(0.288 \mathrm{mmol})$ and $89 \mathrm{mg}$ of silver triflate $(0.346 \mathrm{mmol}, 1.2$ equiv) was added $5 \mathrm{~mL}$ of vinyl acetate and $5 \mathrm{~mL}$ of chloroform, and the reaction mixture was stirred for $20 \mathrm{~h}$ at $\mathrm{rt}$ under dark conditions. The resulting mixture was filtered through Celite ${ }^{\circledR}$, and the filter cake was washed with $\mathrm{CH}_{2} \mathrm{Cl}_{2}$. The conbined organic portions were washed with $1 \mathrm{~N} \mathrm{HCl}$ aq and twice with $\mathrm{H}_{2} \mathrm{O}$. The resulting organic portion was then quickly washed with $1 \mathrm{~N}$ $\mathrm{NaOH}$ aq and twice with $\mathrm{H}_{2} \mathrm{O}$. The mixture was dried over $\mathrm{MgSO}_{4}$, filtered, and evaporated to dryness. The resulting solid was dissolved in $5 \mathrm{~mL}$ of $\mathrm{CH}_{2} \mathrm{Cl}_{2}$ and $20 \mathrm{~mL}$ of pentane was slowly added to the solution. The suspension was filtered to remove the precipitate and the volatile materials were removed in vacuo to afford $\mathbf{2 b}$ as a yellow powder $(80 \mathrm{mg}, 0.146$ mmol, 51\% yield). ${ }^{1} \mathrm{H}$ NMR $\left(\mathrm{CDCl}_{3}\right): \delta 0.77(\mathrm{t}, J=7.2 \mathrm{~Hz}, 3 \mathrm{H}), 1.07-1.17(\mathrm{~m}, 1 \mathrm{H})$, 1.38-1.47 (m, 1H), 2.22 (s, 1H), 4.87 (ddd, $J=7.3,6.4,2.3 \mathrm{~Hz}, 1 \mathrm{H}$ ), 7.22 (ddd, $J=10.6$, 7.6, 1.2 Hz, 1H), 7.37 (dddd, $J=7.6,7.6,1.2,1.2 \mathrm{~Hz}, 1 \mathrm{H}), 7.46-7.61(\mathrm{~m}, 9 \mathrm{H}), 7.76-7.81(\mathrm{~m}$, $2 \mathrm{H}), 8.24(\mathrm{ddd}, J=7.6,4.3,1.2 \mathrm{~Hz}, 1 \mathrm{H}) ;{ }^{31} \mathrm{P}$ NMR $\left(\mathrm{CDCl}_{3}\right): \delta 25.4$; HRMS-FAB $(\mathrm{m} / \mathrm{z})$ : $\left[\mathrm{M}^{+}\right]$calcd for $\mathrm{C}_{23} \mathrm{H}_{23} \mathrm{O}_{5} \mathrm{PPdS}, 547.0057$; found, 547.0045. 
General Procedures of Copolymerization of Vinyl Acetate and Carbon Monoxide Catalyzed by a Pd complex and 1 (Table 1, entry 1-6). To a $50 \mathrm{~mL}$-autoclave containing Pd complex (0.010 mmol), $1(0.012 \mathrm{mmol})$ and a stir bar was transferred $2.5 \mathrm{~mL}$ of vinyl acetate under argon atmosphere. The mixture was stirred at $\mathrm{rt}$ for $15 \mathrm{~min}$ and charged with carbon monoxide. The autoclave was heated and the mixture was stirred. After the reaction, the cooled contents of the autoclave were transferred to a $100 \mathrm{~mL}$ round-bottom flask with a minimum amount of $\mathrm{CH}_{2} \mathrm{Cl}_{2}$ and volatile materials were removed on a rotary evaporator. The remaining solid was dried under vacuum to afford the copolymer, which was analyzed without further purification. Yields of the copolymers were determined by subtraction of the weight of added Pd complex and $\mathbf{1}$ from the amount of solid product obtained.

Reprecipitation ( $\times 2$ ) of the crude materials twice from $\mathrm{CH}_{2} \mathrm{Cl}_{2} / \mathrm{MeOH}$ afforded dba-free material of the alternating copolymers $(210 \mathrm{mg}$, for entry 1$)$.

General Procedures of Copolymerization of Vinyl Acetate and Carbon Monoxide Catalyzed by 2b (Table 1, entry 7-9). To a $50 \mathrm{~mL}$-autoclave containing $\mathbf{2 b}(0.010 \mathrm{mmol})$, galvinoxyl $(0.050 \mathrm{mmol}$, if added), and a stir bar was transferred $2.5 \mathrm{~mL}$ of vinyl acetate under argon atomosphere. The mixture was stirred at $\mathrm{rt}$ for $15 \mathrm{~min}$ and charged with carbon monoxide. The autoclave was heated and the mixture was stirred. After the reaction, the cooled contents of the autoclave were transferred to a $100 \mathrm{~mL}$ round-bottom flask with a minimum amount of $\mathrm{CH}_{2} \mathrm{Cl}_{2}$ and volatile materials were removed on a rotary evaporator. The remaining solid was dried under vacuum to afford the copolymer, which was analyzed without further purification. Yields of the copolymers were determined by subtraction of the weight of added $\mathbf{2 b}$ (and galvinoxyl) from the amount of solid product obtained. 
Table S1. Copolymerization of vinyl acetate with carbon monoxide.

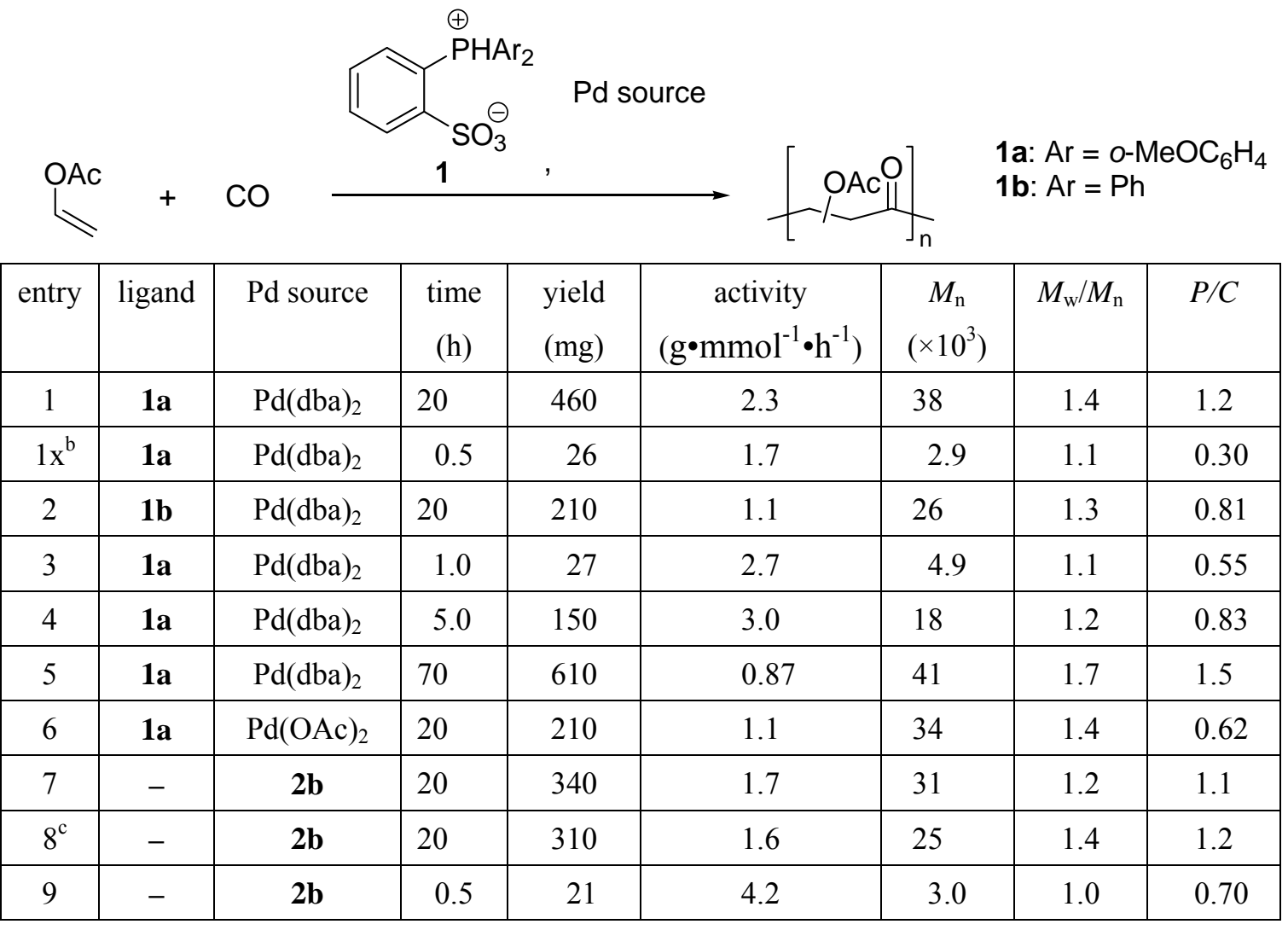

${ }^{\mathrm{a}}$ Conditions: $0.010 \mathrm{mmol}$ of $\mathrm{Pd}$ source, $0.012 \mathrm{mmol}$ of ligand, $6.0 \mathrm{MPa}$ of CO pressure, $2.5 \mathrm{~mL}$ of vinyl acetate, $70{ }^{\circ} \mathrm{C} .{ }^{\mathrm{b}} 0.036 \mathrm{mmol}$ of $\mathbf{1 a}$ and $0.030 \mathrm{mmol}$ of $\mathrm{Pd}(\mathrm{dba})_{2}$ were used. ${ }^{\mathrm{c}} 0.050 \mathrm{mmol}$ of galvinoxyl was added. 


\section{Characterization of Vinyl Acetate/Carbon Monoxide Copolymers}

${ }^{1} \mathrm{H}$ NMR $\left(\mathrm{CDCl}_{3}, 500 \mathrm{MHz}\right): 1.99-2.26\left(-\mathrm{OCOCH}_{3}\right), 2.76-3.51\left(-\mathrm{CH}_{2}-\right), 5.24-5.76$ $(-\mathrm{CH}(\mathrm{OAc})-)$.

${ }^{13} \mathrm{C} \mathrm{NMR}\left(\mathrm{CDCl}_{3}, 126 \mathrm{MHz}\right): 20.2-20.7\left(-\mathrm{OCOCH}_{3}\right), 38.9-40.6,42.4-44.2\left(-\mathrm{CH}_{2}-\right)$, 70.2-71.4, 72.7-73.5 (-CH(OAc)-), 169.8-170.3 $\left(-\mathrm{OCOCH}_{3}\right), 201.1-201.5,201.6-203.2$ $(-\mathrm{CO}-)$.

Results of HH-COSY, HMQC, and DEPT experiments are consistent with the peak assiginment shown above. Carbons on the polymer backbones showed distinct splitting of peaks. The minor regions of the peaks of the methylenes (42.4-44.2 ppm), the methynes (70.2-71.4 ppm), and the carbonyl carbons (201.1-201.5 ppm) account for 25, 25, and $13 \%$ of the total regions of each carbon, respectively. The observed broadening and distinct splitting of the peaks indicate that the copolymerization does not proceed in a complete regioregular fashion. If the splitting arises from misinsertion (1,2-insertion, instead of 2,1-insertion) of vinyl acetate, head-to-tail selectivity of the copolymerization (entry 1) should be $87 \%$.

Initiating chain ends were determined by ${ }^{1} \mathrm{H}$ NMR and HH-COSY experiments. For this purpose, alternating copolymer with $M_{\mathrm{n}}$ of $2900\left(M_{\mathrm{w}} / M_{\mathrm{n}} 1.1\right)$ was prepared by reaction of $7.5 \mathrm{~mL}$ of vinyl acetate under $6.0 \mathrm{MPa}$ of carbon monoxide in the presence of $0.030 \mathrm{mmol}$ of $\operatorname{Pd}(\mathrm{dba})_{2}$ and $0.036 \mathrm{mmol}$ of $1 \mathbf{a}$ (Table S1, entry $1 \mathrm{x}$ ). The obtained copolymers were further analyzed by MALDI-TOF MS to show signals with intervals of 114. The major series of peaks on the MALDI-TOF mass spectrum of the copolymer obtained with $\mathbf{2 b}$ (Table 1, entry 9) were assigned to a completely alternating copolymer initiated by $\mathrm{CO}$ insertion to 1-acetoxypropylpalladium and terminated by protonolysis of alkylpalladium resulting from the last insertion of vinyl acetate (Figure S13). The signals of the copolymers on the MALDI-TOF mass spectrum appeared as sodium adducts and potassium adducts, which are commonly observed for this analysis method.

Structures of terminating chain ends of the copolymers have not been identified, but presence of palladium at the chain ends is expected because of the remarkable stability of 1-acetoxypropyl chelates such as $\mathbf{2 b}$ under acid/base conditions.

The number of produced polymer chains per catalysts for each entry $(P / C)$ was calculated and listed on Table S1. Assuming that the actual molecular weights of the copolymers are not much different from $M_{\mathrm{n}}$ calculated based on polystyrene standards, the fact that $P / C$ s were close to 1 indicate that each catalyst molecule worked to produce about 
one polymer chain. Initiation for the system with $\operatorname{Pd}(\mathrm{dba})_{2}$ and 1a may not be as efficient as $\mathbf{2 b}$, based on relatively small $P / C$ s for entries with short reaction times. Chain transfer may not be frequently occurring, because of the lack of significant increase in $P / C$ for entries with relatively longer reaction times.

The alternating copolymer of vinyl acetate and carbon monoxide may form a spiroketal structure, a structural isomer of the $\gamma$-polyketone, upon stading in solution for a long time, as observed for other $\gamma$-polyketones.

\section{References}

1. E. Drent, R. van Dijk, R. van Ginkel, B. van Oort, and R. I. Pugh, Chem. Commun., 2002, 964-965.

2. Eur. Pat., 0280 380, 1988.

3. T. Kochi, K. Yoshimura, K. Nozaki, Dalton Trans. 2006, 25-27. 


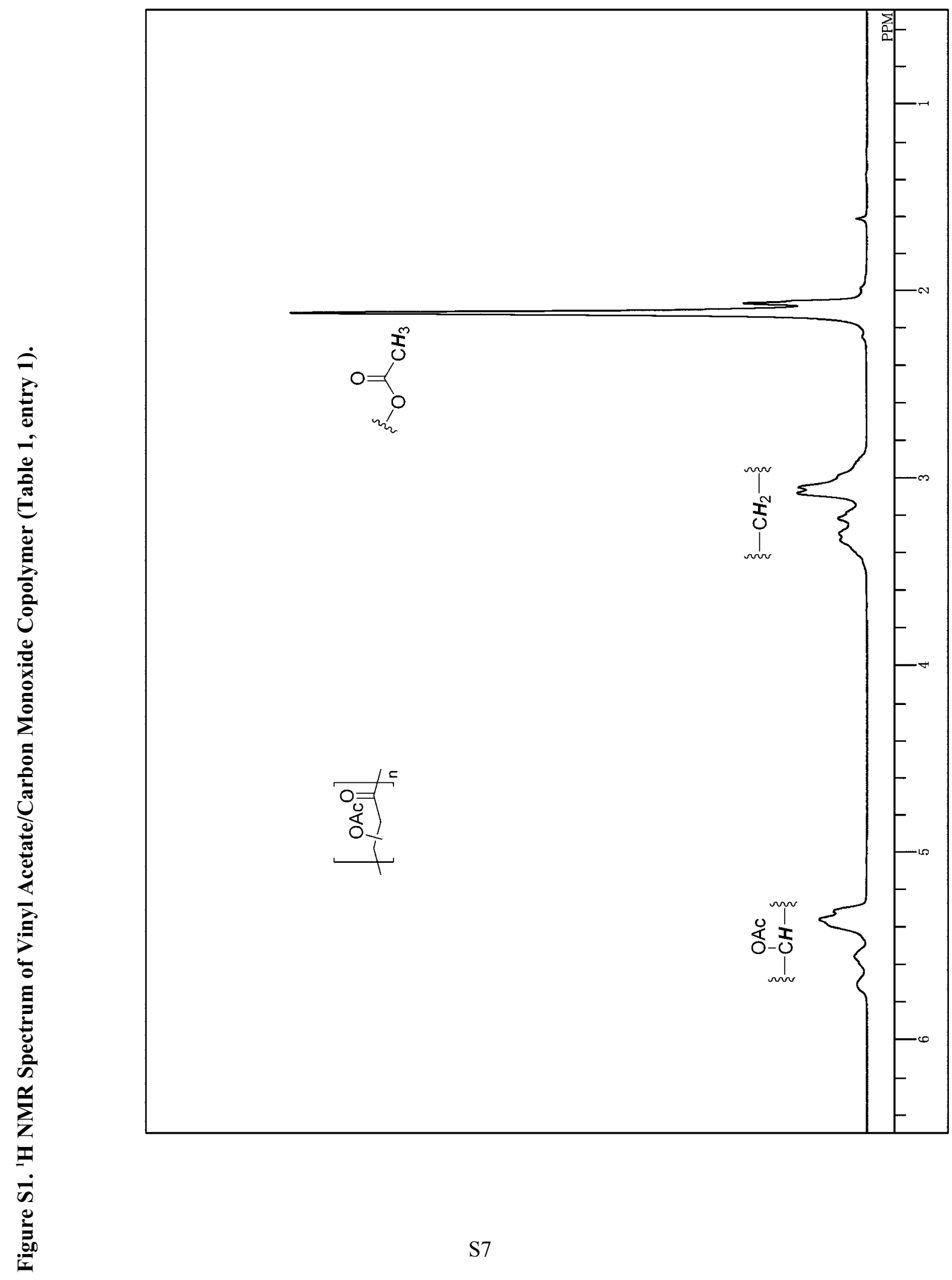




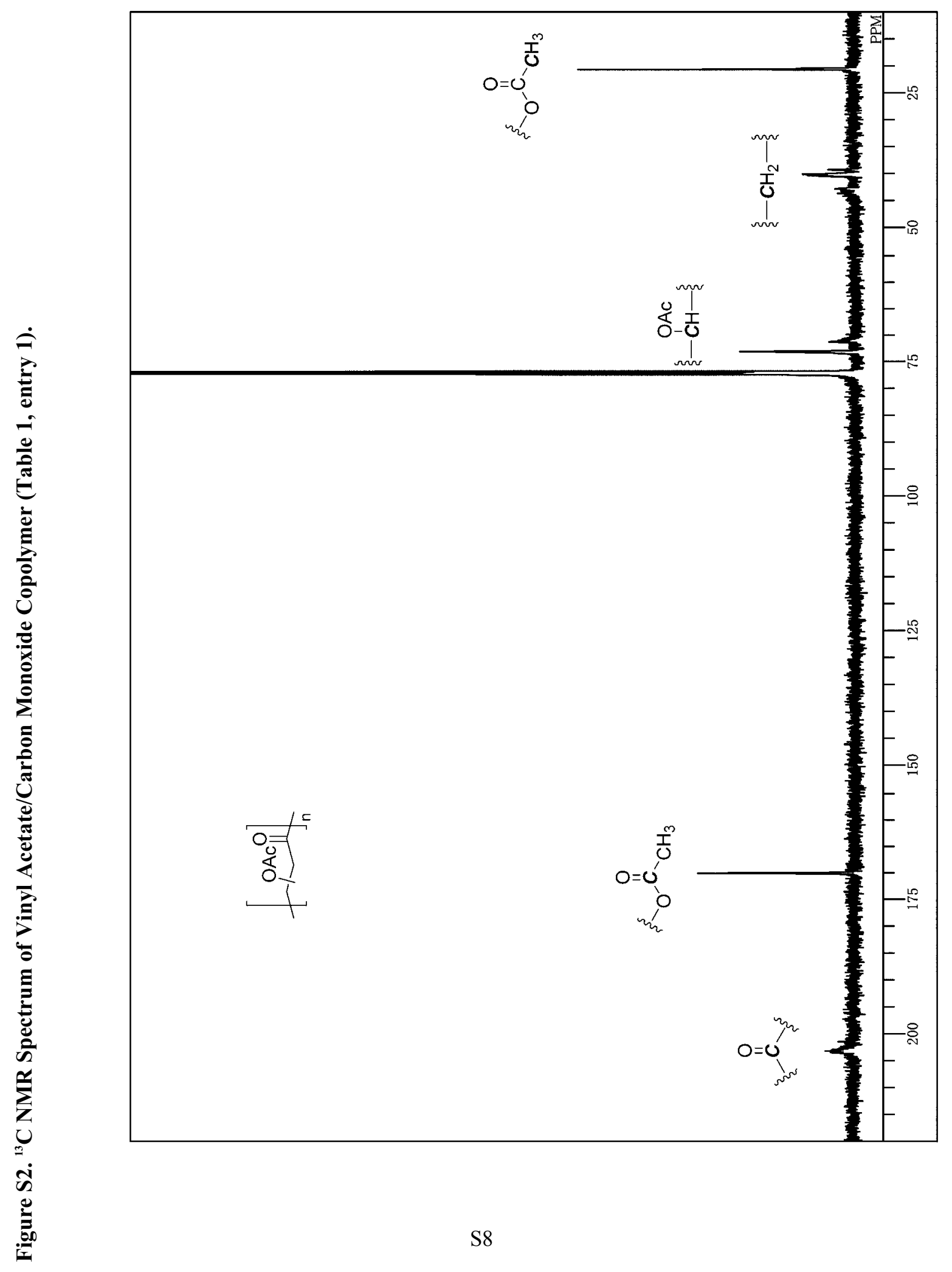




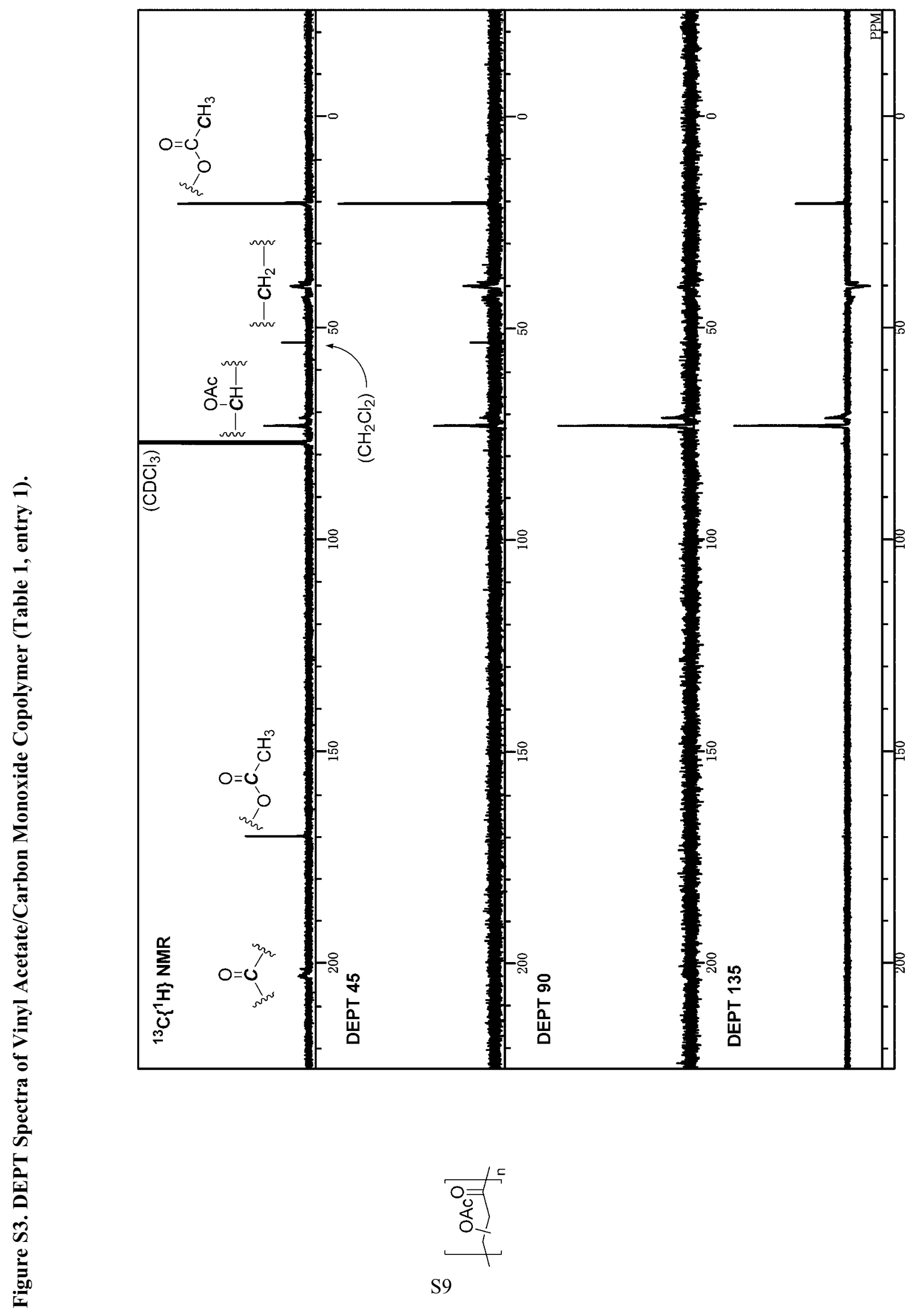




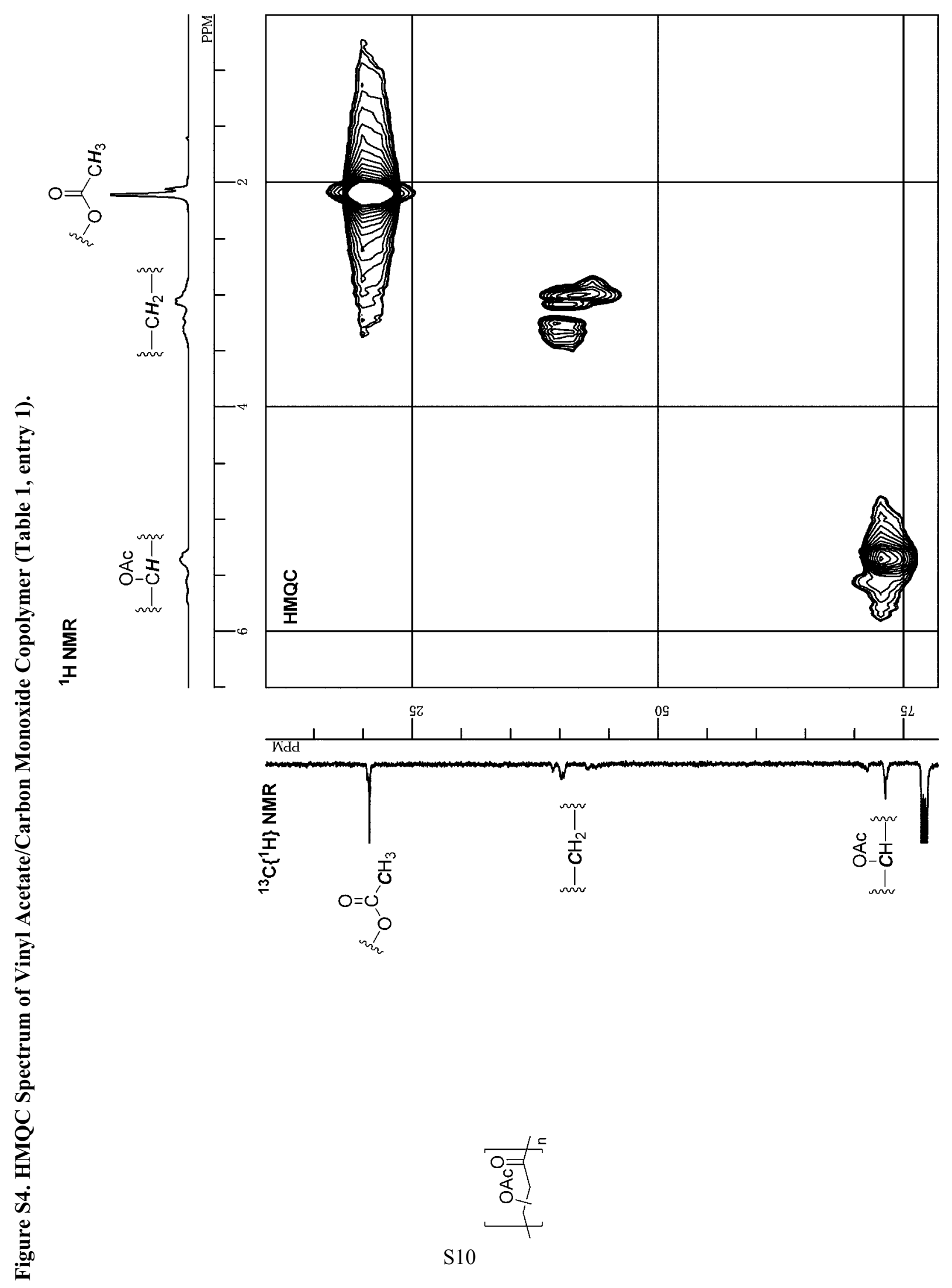




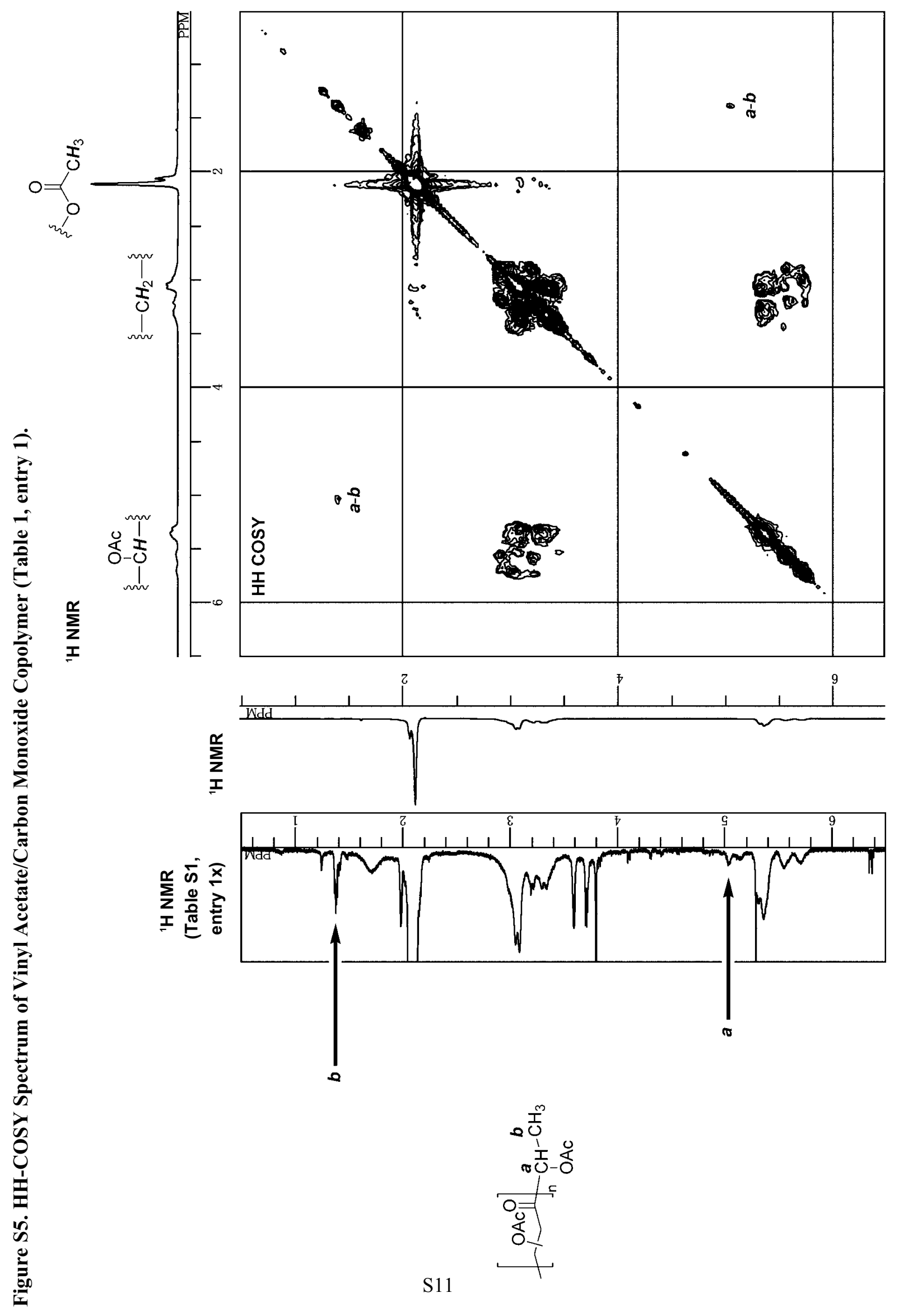




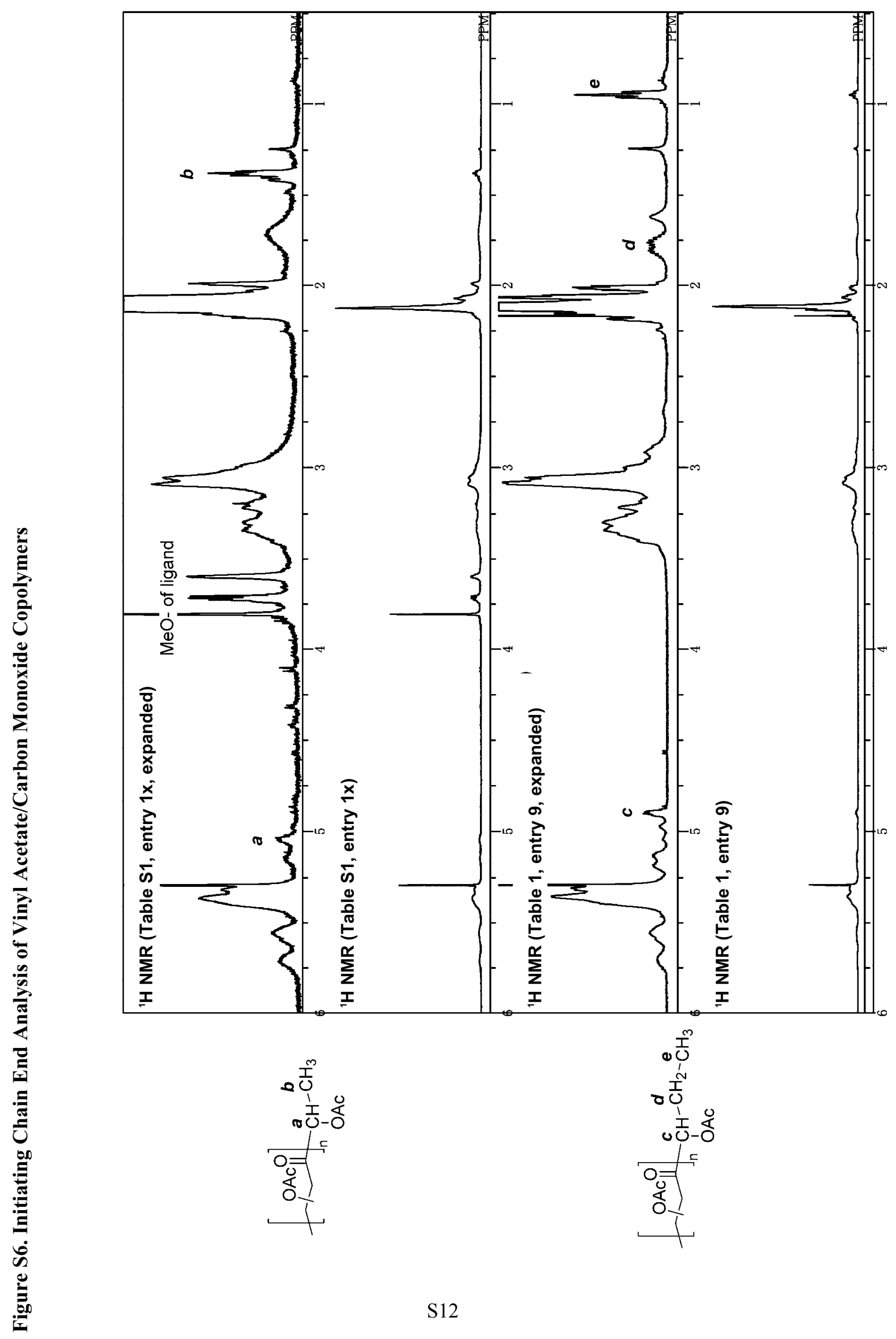



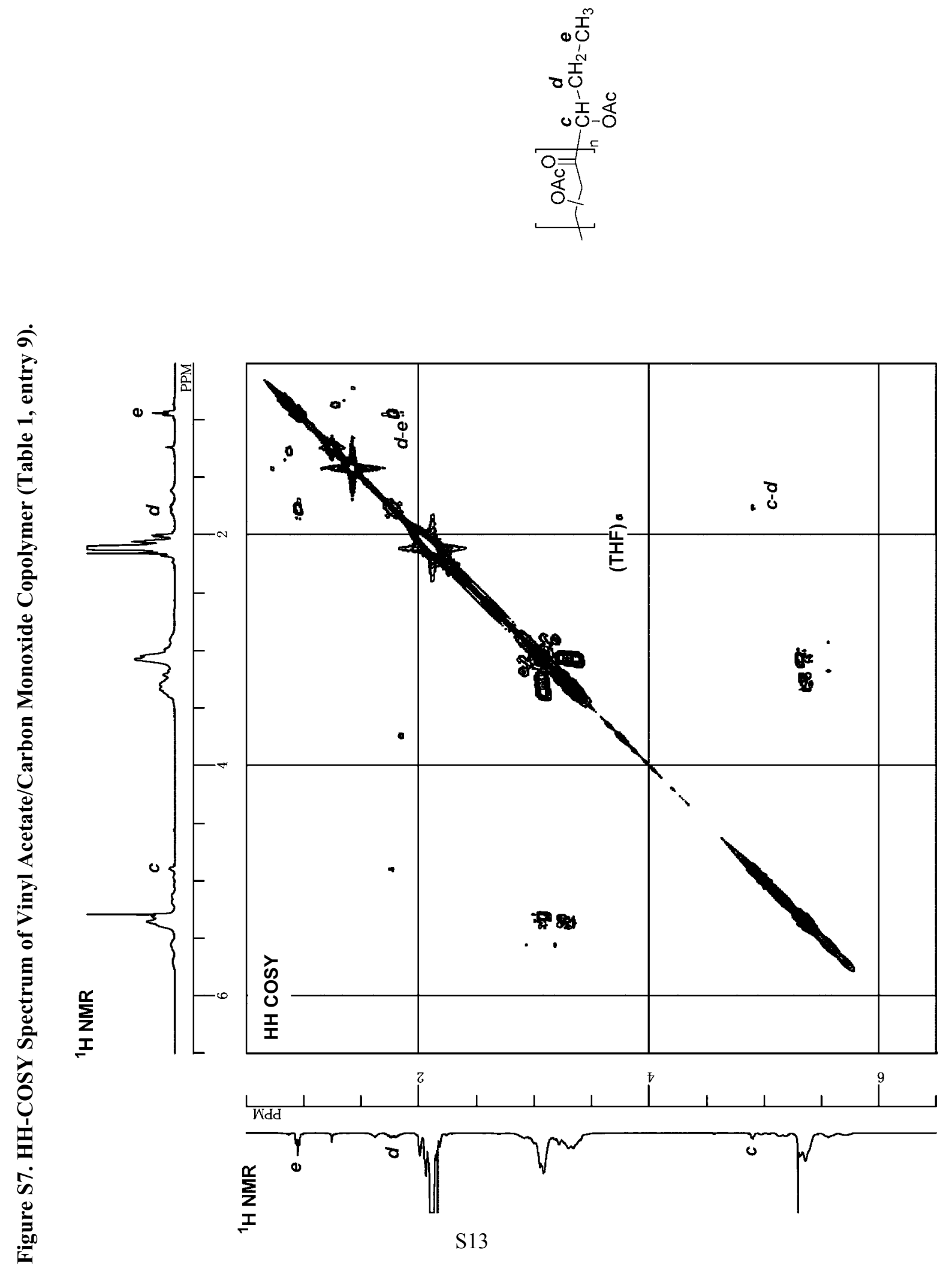
Figure S8. MALDI-TOF MS of Vinyl Acetate/Carbon Monoxide Copolymer.

Table 1, entry 9

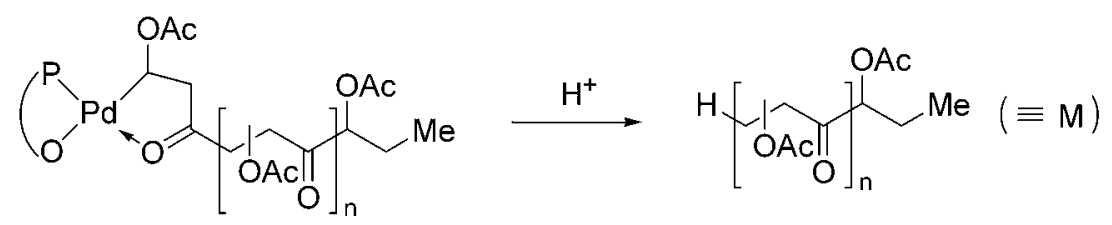

$$
\bigcirc:[\mathrm{M}+\mathrm{Na}]^{+}
$$

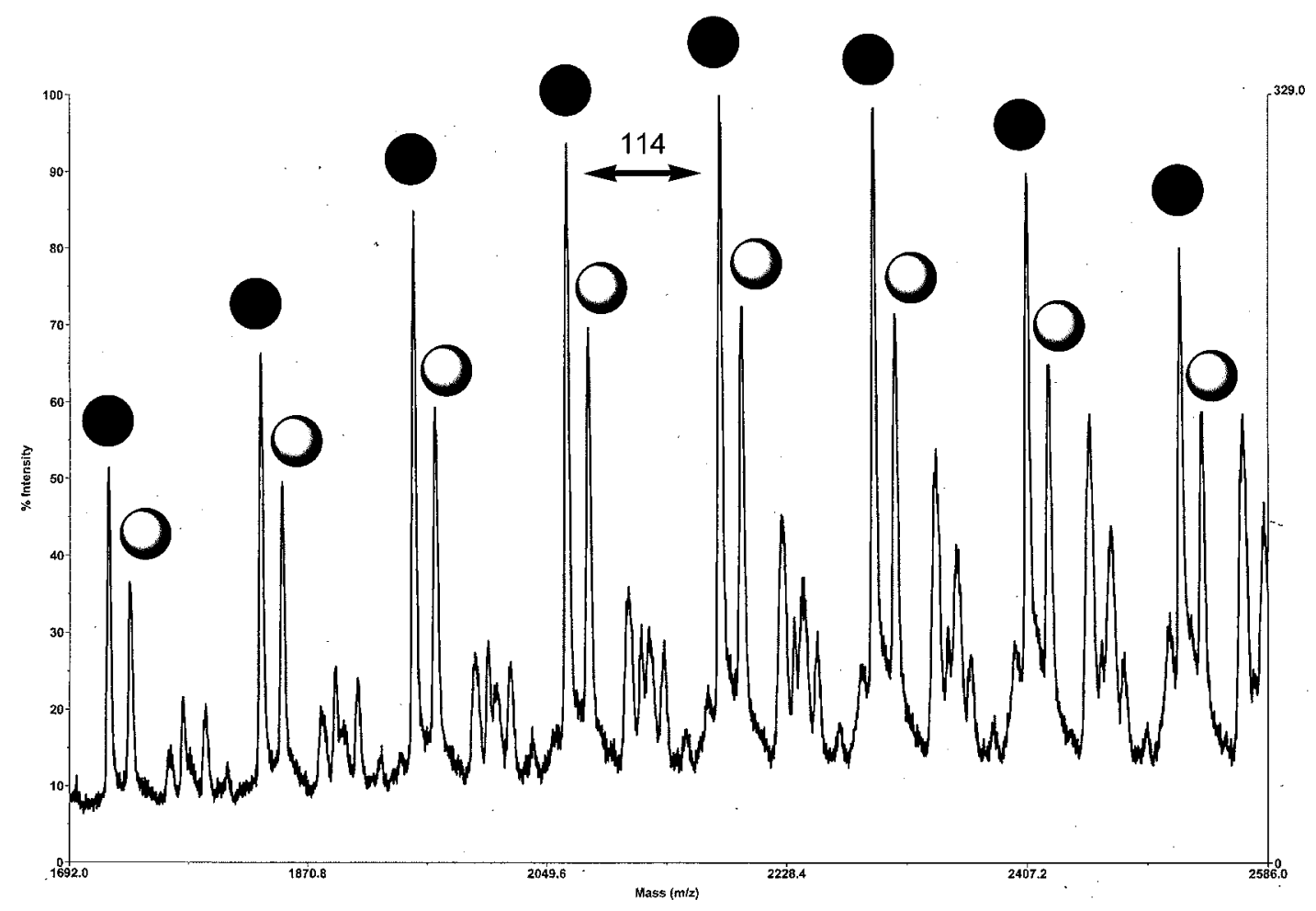




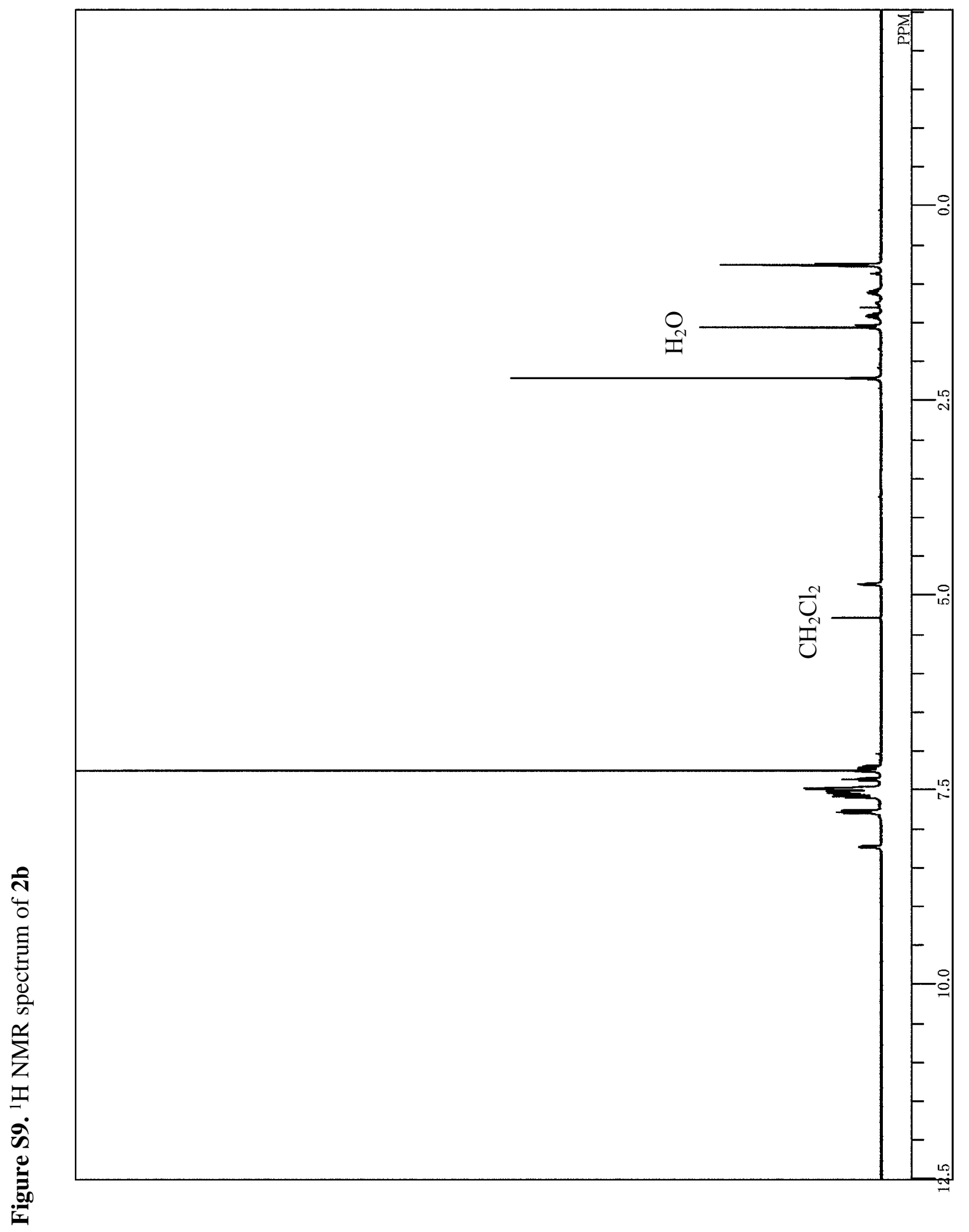

S15 
Figure S10. X-ray structure of $\mathbf{2 b}$.

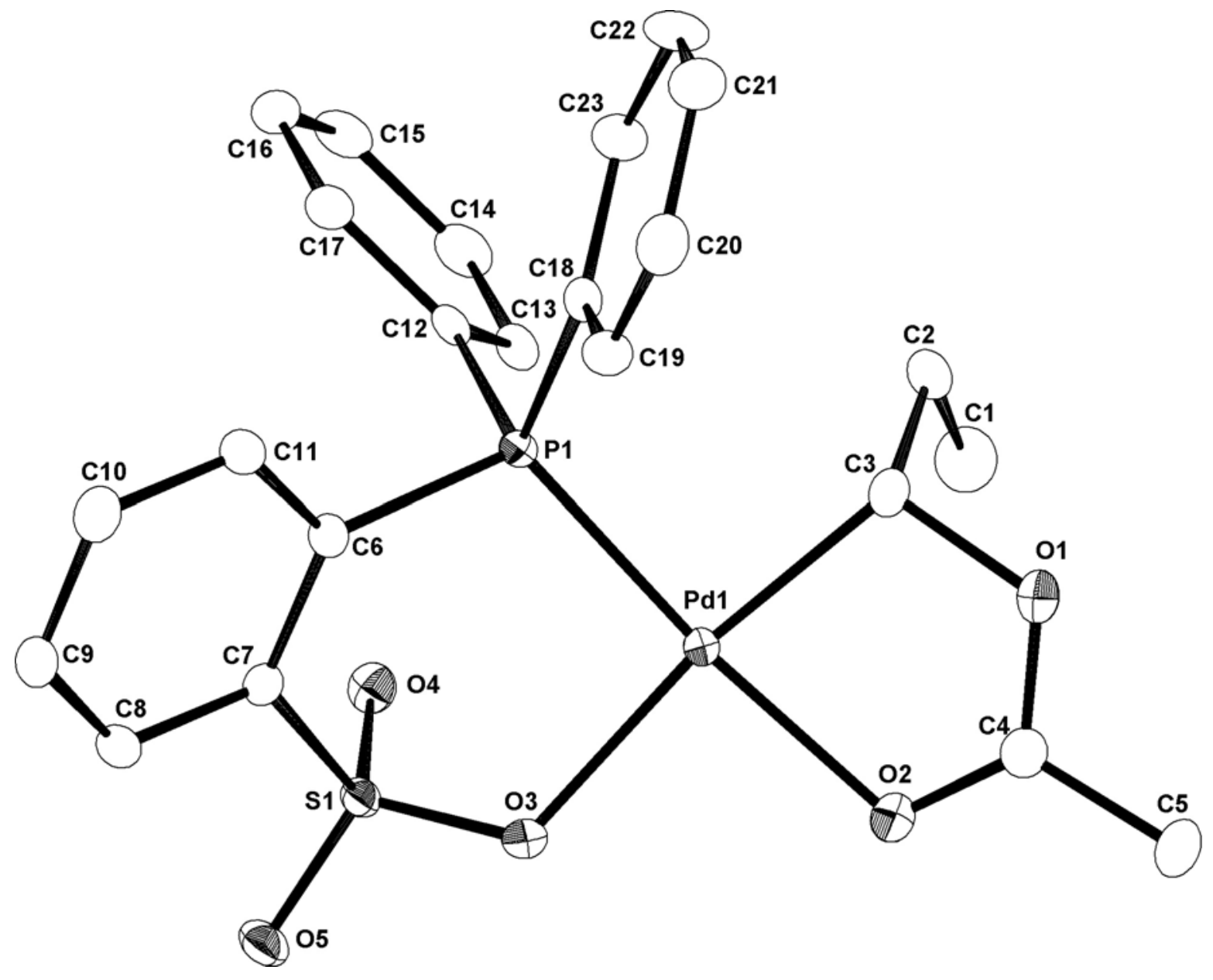


Table S2. Crystal data and structure refinement for $\mathbf{2 b}$.

\begin{tabular}{|c|c|}
\hline Empirical formula & $\mathrm{C}_{23} \mathrm{H}_{23} \mathrm{O}_{5} \mathrm{PPdS}$ \\
\hline Formula weight & 548.84 \\
\hline Temperature & $103(2) \mathrm{K}$ \\
\hline Crystal color, habit & yellow, block \\
\hline Crystal dimensions & $0.60 \times 0.40 \times 0.30 \mathrm{~mm}$ \\
\hline Crystal system & monoclinic \\
\hline Space group & $P 2_{1} / \mathrm{c}$ \\
\hline $\mathrm{a}(\AA)$ & $9.2821(9)$ \\
\hline $\mathrm{b}(\AA)$ & $17.5262(11)$ \\
\hline c $(\AA)$ & $13.8150(10)$ \\
\hline$\beta\left(^{\circ}\right)$ & $101.4069(14)$ \\
\hline$V\left(\AA^{3}\right)$ & $2203.0(3)$ \\
\hline $\mathrm{Z}$ & 4 \\
\hline Density (calculated) & $1.655 \mathrm{mg} / \mathrm{m}^{3}$ \\
\hline $\mathrm{F}(000)$ & 1112 \\
\hline Absorption coefficient & $1.043 \mathrm{~mm}^{-1}$ \\
\hline Diffractometer & Rigaku Mercury CCD \\
\hline \multirow[t]{2}{*}{ Radiation } & $\operatorname{MoK} \alpha(\lambda=0.71070 \AA)$ \\
\hline & graphite monochromated \\
\hline Theta range for data collection & 3.01 to $25.00^{\circ}$. \\
\hline Index ranges & $-11<=\mathrm{h}<=10,-20<=\mathrm{k}<=19,-16<=1<=16$ \\
\hline Reflections collected & 13888 \\
\hline Independent reflections & $3833[\mathrm{R}(\mathrm{int})=0.0214]$ \\
\hline Completeness to theta $=25.00^{\circ}$ & $98.8 \%$ \\
\hline Absorption correction & Semi-empirical from equivalents \\
\hline Max. and min. transmission & 0.7301 and 0.5619 \\
\hline Refinement method & Full-matrix least-squares on $\mathrm{F}^{2}$ \\
\hline Data / restraints / parameters & $3833 / 0 / 282$ \\
\hline Goodness-of-fit on $\mathrm{F}^{2}$ & 1.072 \\
\hline Final $R$ indices $[\mathrm{I}>2 \operatorname{sigma}(\mathrm{I})]$ & $\mathrm{R} 1=0.0241, \mathrm{wR} 2=0.0603$ \\
\hline $\mathrm{R}$ indices (all data) & $\mathrm{R} 1=0.0252, \mathrm{wR} 2=0.0610$ \\
\hline Largest diff. peak and hole & 0.661 and -0.539 e. $\AA^{-3}$ \\
\hline
\end{tabular}

${ }^{\mathrm{a}}$ Goodness of Fit $=\left\{\Sigma\left[\mathrm{w}\left(\left|\mathrm{F}_{\mathrm{o}}\right|-\left|\mathrm{F}_{\mathrm{c}}\right|\right)^{2}\right] /\left(\mathrm{N}_{\mathrm{obs}}-\mathrm{N}_{\text {parameters }}\right)\right\}^{1 / 2}$

${ }^{\mathrm{b}} \mathrm{R}=\Sigma|| \mathrm{F}_{\mathrm{o}}|-| \mathrm{F}_{\mathrm{c}}|| / \Sigma\left|\mathrm{F}_{\mathrm{o}}\right|, \mathrm{R}_{\mathrm{w}}=\left\{\Sigma\left[\mathrm{w}\left(\left|\mathrm{F}_{\mathrm{o}}\right|-\left|\mathrm{F}_{\mathrm{c}}\right|\right)^{2}\right] / \Sigma\left[\mathrm{wF}_{\mathrm{o}}{ }^{2}\right]\right\}^{1 / 2}$ 


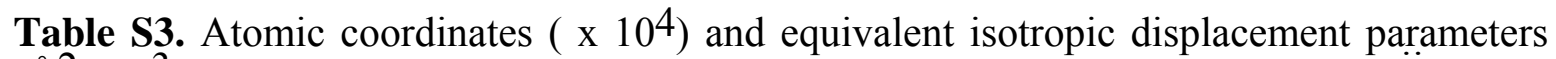
$\left(\AA^{2} \times 10^{3}\right)$ for $\mathbf{2 b}$. U(eq) is defined as one third of the trace of the orthogonalized $U^{i j}$ tensor.

\begin{tabular}{|c|c|c|c|c|}
\hline & $\mathrm{x}$ & $\mathrm{y}$ & $\mathrm{z}$ & $\mathrm{U}(\mathrm{eq})$ \\
\hline$\overline{P d(1)}$ & $5091(1)$ & 1451(1) & $6746(1)$ & $11(1)$ \\
\hline$C(1)$ & $5851(3)$ & $2252(2)$ & $9034(2)$ & $28(1)$ \\
\hline$C(2)$ & $6294(3)$ & $1426(1)$ & $8938(2)$ & $20(1)$ \\
\hline $\mathrm{C}(3)$ & $6656(3)$ & 1241(1) & $7939(2)$ & $17(1)$ \\
\hline $\mathrm{O}(1)$ & $7932(2)$ & $1735(1)$ & $7850(1)$ & $19(1)$ \\
\hline $\mathrm{C}(4)$ & $7735(3)$ & $2245(1)$ & $7150(2)$ & $15(1)$ \\
\hline $\mathrm{O}(2)$ & $6564(2)$ & $2315(1)$ & $6549(1)$ & $17(1)$ \\
\hline$C(5)$ & $9055(3)$ & $2720(1)$ & $7111(2)$ & $20(1)$ \\
\hline $\mathrm{P}(1)$ & $3809(1)$ & $429(1)$ & $7023(1)$ & $11(1)$ \\
\hline$C(6)$ & $2763(2)$ & $58(1)$ & $5852(2)$ & $13(1)$ \\
\hline$C(7)$ & $2038(2)$ & $546(1)$ & $5102(2)$ & $11(1)$ \\
\hline$S(1)$ & 2084(1) & $1556(1)$ & $5247(1)$ & $12(1)$ \\
\hline $\mathrm{O}(3)$ & $3664(2)$ & $1758(1)$ & $5388(1)$ & $16(1)$ \\
\hline $\mathrm{O}(4)$ & $1496(2)$ & $1715(1)$ & $6122(1)$ & $16(1)$ \\
\hline $\mathrm{O}(5)$ & $1263(2)$ & 1873(1) & $4339(1)$ & $19(1)$ \\
\hline $\mathrm{C}(8)$ & $1248(3)$ & $246(1)$ & $4225(2)$ & $14(1)$ \\
\hline$C(9)$ & $1159(3)$ & $-536(1)$ & $4080(2)$ & $16(1)$ \\
\hline$C(10)$ & 1839(3) & $-1020(1)$ & $4817(2)$ & $17(1)$ \\
\hline $\mathrm{C}(11)$ & 2643(3) & $-726(1)$ & $5692(2)$ & $15(1)$ \\
\hline$C(12)$ & $2461(2)$ & $514(1)$ & $7808(2)$ & $13(1)$ \\
\hline$C(13)$ & $2255(3)$ & $1196(1)$ & $8274(2)$ & $15(1)$ \\
\hline$C(14)$ & $1216(3)$ & $1244(2)$ & $8871(2)$ & $19(1)$ \\
\hline$C(15)$ & $376(3)$ & $614(2)$ & 8994(2) & $20(1)$ \\
\hline$C(16)$ & $561(3)$ & $-68(1)$ & $8526(2)$ & $17(1)$ \\
\hline$C(17)$ & $1603(3)$ & $-119(1)$ & $7934(2)$ & $15(1)$ \\
\hline$C(18)$ & $4968(3)$ & $-364(1)$ & $7548(2)$ & $14(1)$ \\
\hline$C(19)$ & $5912(3)$ & $-687(1)$ & $6990(2)$ & $17(1)$ \\
\hline$C(20)$ & $6853(3)$ & $-1273(2)$ & $7379(2)$ & $21(1)$ \\
\hline$C(21)$ & $6883(3)$ & $-1532(1)$ & $8326(2)$ & $23(1)$ \\
\hline$C(22)$ & 5974(3) & $-1210(2)$ & $8888(2)$ & $25(1)$ \\
\hline $\mathrm{C}(23)$ & $5012(3)$ & $-624(1)$ & $8509(2)$ & $19(1)$ \\
\hline
\end{tabular}


Table S4. Bond lengths $[\AA]$ and angles $\left[{ }^{\circ}\right]$ for $\mathbf{2 b}$.

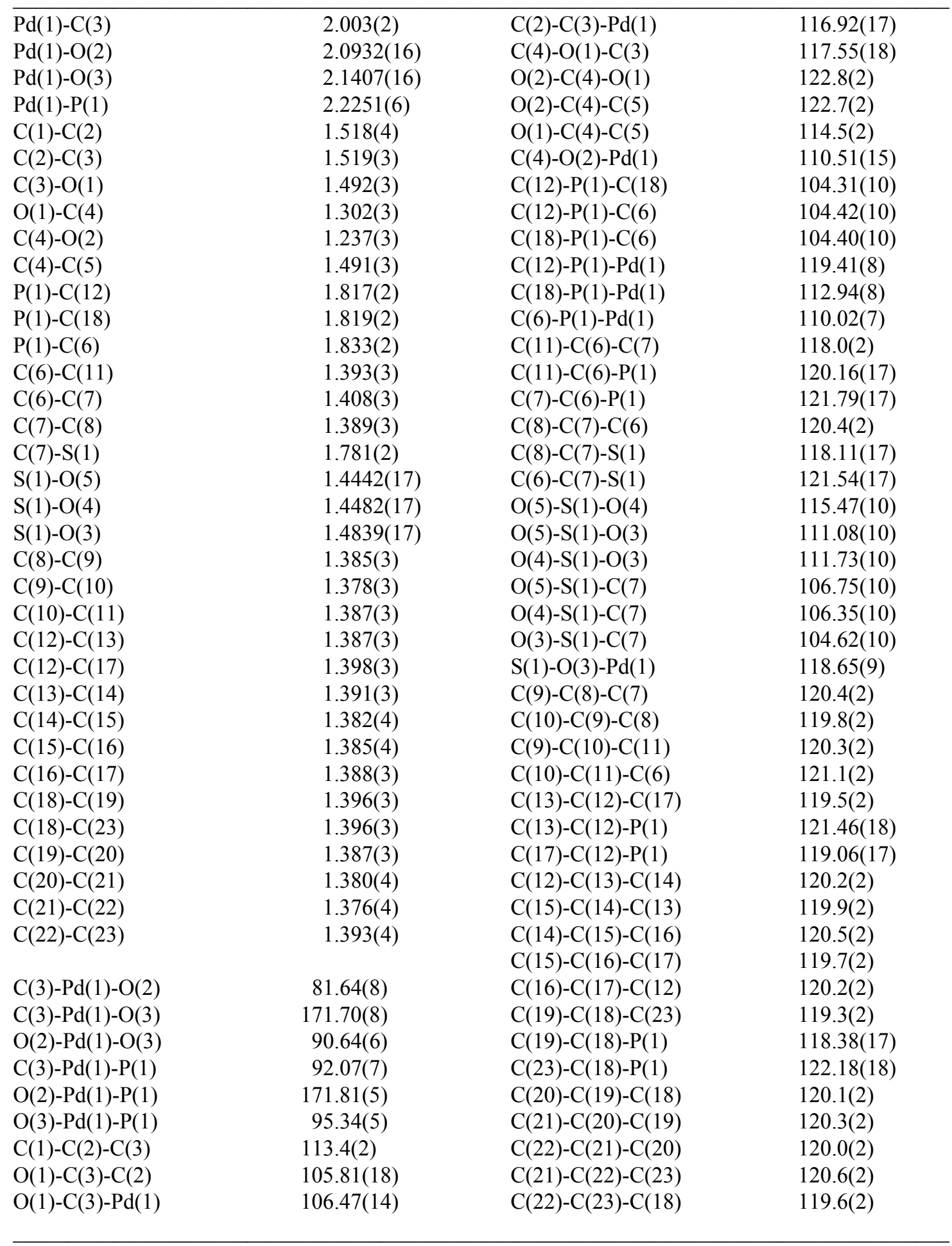


Table S5. Anisotropic displacement parameters $\left(\AA^{2} \times 10^{3}\right)$ for $2 \mathbf{b}$. The anisotropic displacement factor exponent takes the form: $-2 \pi^{2}\left[\mathrm{~h}^{2} \mathrm{a} * 2 \mathrm{U}^{11}+\ldots+2 \mathrm{~h} \mathrm{k} \mathrm{a}^{*} \mathrm{~b}^{*} \mathrm{U}^{12}\right]$.

\begin{tabular}{|c|c|c|c|c|c|c|}
\hline & $\mathrm{U}^{11}$ & $\mathrm{U}^{22}$ & $\mathrm{U}^{33}$ & $\mathrm{U}^{23}$ & $\mathrm{U}^{13}$ & $\mathrm{U}^{12}$ \\
\hline $\operatorname{Pd}(1)$ & 11(1) & $12(1)$ & $10(1)$ & $0(1)$ & 1(1) & $-2(1)$ \\
\hline$C(1)$ & $33(2)$ & $26(1)$ & $25(1)$ & $-6(1)$ & $6(1)$ & $-3(1)$ \\
\hline$C(2)$ & $18(1)$ & $26(1)$ & $13(1)$ & $0(1)$ & $0(1)$ & $-4(1)$ \\
\hline$C(3)$ & $13(1)$ & $18(1)$ & $18(1)$ & $1(1)$ & 1(1) & $-4(1)$ \\
\hline $\mathrm{O}(1)$ & $14(1)$ & $22(1)$ & 21(1) & $1(1)$ & $1(1)$ & $-4(1)$ \\
\hline C(4) & $16(1)$ & $14(1)$ & $15(1)$ & $-3(1)$ & $5(1)$ & $0(1)$ \\
\hline $\mathrm{O}(2)$ & $16(1)$ & $17(1)$ & $18(1)$ & $0(1)$ & $3(1)$ & $-3(1)$ \\
\hline$C(5)$ & $16(1)$ & 19(1) & $24(1)$ & $-3(1)$ & $5(1)$ & $-5(1)$ \\
\hline $\mathrm{P}(1)$ & 11(1) & 11(1) & $10(1)$ & $1(1)$ & $1(1)$ & $0(1)$ \\
\hline$C(6)$ & 11(1) & $15(1)$ & $12(1)$ & $-1(1)$ & $3(1)$ & $-1(1)$ \\
\hline$C(7)$ & 11(1) & $12(1)$ & $12(1)$ & $-1(1)$ & $4(1)$ & $0(1)$ \\
\hline $\mathrm{S}(1)$ & $14(1)$ & 11(1) & 11(1) & $1(1)$ & $-1(1)$ & $0(1)$ \\
\hline $\mathrm{O}(3)$ & $17(1)$ & $15(1)$ & $14(1)$ & $3(1)$ & $2(1)$ & $-3(1)$ \\
\hline $\mathrm{O}(4)$ & $18(1)$ & $14(1)$ & $17(1)$ & $-3(1)$ & $4(1)$ & $0(1)$ \\
\hline $\mathrm{O}(5)$ & $22(1)$ & $15(1)$ & $16(1)$ & $3(1)$ & $-4(1)$ & $1(1)$ \\
\hline $\mathrm{C}(8)$ & $13(1)$ & $18(1)$ & $12(1)$ & $1(1)$ & $3(1)$ & $1(1)$ \\
\hline $\mathrm{C}(9)$ & $15(1)$ & $20(1)$ & $13(1)$ & $-3(1)$ & $3(1)$ & $-3(1)$ \\
\hline$C(10)$ & $18(1)$ & $13(1)$ & 19(1) & $-6(1)$ & $5(1)$ & $-1(1)$ \\
\hline $\mathrm{C}(11)$ & $14(1)$ & $14(1)$ & $15(1)$ & $1(1)$ & $2(1)$ & $1(1)$ \\
\hline $\mathrm{C}(12)$ & $10(1)$ & $17(1)$ & $9(1)$ & $2(1)$ & $-1(1)$ & $1(1)$ \\
\hline$C(13)$ & $14(1)$ & $16(1)$ & $13(1)$ & $0(1)$ & $-1(1)$ & $1(1)$ \\
\hline$C(14)$ & $22(1)$ & $20(1)$ & $15(1)$ & $-1(1)$ & $2(1)$ & $6(1)$ \\
\hline$C(15)$ & $18(1)$ & $29(1)$ & $13(1)$ & $4(1)$ & $4(1)$ & $6(1)$ \\
\hline$C(16)$ & $16(1)$ & $20(1)$ & $16(1)$ & $4(1)$ & $4(1)$ & $-1(1)$ \\
\hline$C(17)$ & $17(1)$ & $15(1)$ & $14(1)$ & $1(1)$ & $2(1)$ & $1(1)$ \\
\hline$C(18)$ & $12(1)$ & $14(1)$ & $14(1)$ & $1(1)$ & $0(1)$ & $-1(1)$ \\
\hline$C(19)$ & $17(1)$ & $18(1)$ & $17(1)$ & $3(1)$ & $3(1)$ & $1(1)$ \\
\hline$C(20)$ & $14(1)$ & 21(1) & $28(1)$ & $-2(1)$ & $6(1)$ & $2(1)$ \\
\hline $\mathrm{C}(21)$ & $18(1)$ & $19(1)$ & $32(1)$ & $10(1)$ & $1(1)$ & $4(1)$ \\
\hline $\mathrm{C}(22)$ & $22(1)$ & $31(2)$ & $22(1)$ & $15(1)$ & $4(1)$ & $5(1)$ \\
\hline$C(23)$ & 19(1) & $22(1)$ & $17(1)$ & $4(1)$ & $4(1)$ & $2(1)$ \\
\hline
\end{tabular}


Table S6. Torsion angles $\left[{ }^{\circ}\right]$ for $\mathbf{2 b}$.

\begin{tabular}{lc}
\hline $\mathrm{C}(1)-\mathrm{C}(2)-\mathrm{C}(3)-\mathrm{O}(1)$ & \\
$\mathrm{C}(1)-\mathrm{C}(2)-\mathrm{C}(3)-\mathrm{Pd}(1)$ & $61.5(3)$ \\
$\mathrm{O}(2)-\mathrm{Pd}(1)-\mathrm{C}(3)-\mathrm{O}(1)$ & $-56.8(3)$ \\
$\mathrm{O}(3)-\mathrm{Pd}(1)-\mathrm{C}(3)-\mathrm{O}(1)$ & $-8.28(14)$ \\
$\mathrm{P}(1)-\mathrm{Pd}(1)-\mathrm{C}(3)-\mathrm{O}(1)$ & $13.4(7)$ \\
$\mathrm{O}(2)-\mathrm{Pd}(1)-\mathrm{C}(3)-\mathrm{C}(2)$ & $166.47(14)$ \\
$\mathrm{O}(3)-\mathrm{Pd}(1)-\mathrm{C}(3)-\mathrm{C}(2)$ & $109.68(18)$ \\
$\mathrm{P}(1)-\mathrm{Pd}(1)-\mathrm{C}(3)-\mathrm{C}(2)$ & $131.3(5)$ \\
$\mathrm{C}(2)-\mathrm{C}(3)-\mathrm{O}(1)-\mathrm{C}(4)$ & $-75.58(17)$ \\
$\mathrm{Pd}(1)-\mathrm{C}(3)-\mathrm{O}(1)-\mathrm{C}(4)$ & $-116.2(2)$ \\
$\mathrm{C}(3)-\mathrm{O}(1)-\mathrm{C}(4)-\mathrm{O}(2)$ & $8.8(2)$ \\
$\mathrm{C}(3)-\mathrm{O}(1)-\mathrm{C}(4)-\mathrm{C}(5)$ & $-2.4(3)$ \\
$\mathrm{O}(1)-\mathrm{C}(4)-\mathrm{O}(2)-\mathrm{Pd}(1)$ & $179.67(19)$ \\
$\mathrm{C}(5)-\mathrm{C}(4)-\mathrm{O}(2)-\mathrm{Pd}(1)$ & $-5.2(3)$ \\
$\mathrm{C}(3)-\mathrm{Pd}(1)-\mathrm{O}(2)-\mathrm{C}(4)$ & $172.48(17)$ \\
$\mathrm{O}(3)-\mathrm{Pd}(1)-\mathrm{O}(2)-\mathrm{C}(4)$ & $7.96(16)$ \\
$\mathrm{P}(1)-\mathrm{Pd}(1)-\mathrm{O}(2)-\mathrm{C}(4)$ & $-168.99(15)$ \\
$\mathrm{C}(3)-\mathrm{Pd}(1)-\mathrm{P}(1)-\mathrm{C}(12)$ & $-32.1(4)$ \\
$\mathrm{O}(2)-\mathrm{Pd}(1)-\mathrm{P}(1)-\mathrm{C}(12)$ & $88.63(11)$ \\
$\mathrm{O}(3)-\mathrm{Pd}(1)-\mathrm{P}(1)-\mathrm{C}(12)$ & $128.2(3)$ \\
$\mathrm{C}(3)-\mathrm{Pd}(1)-\mathrm{P}(1)-\mathrm{C}(18)$ & $-95.13(9)$ \\
$\mathrm{O}(2)-\mathrm{Pd}(1)-\mathrm{P}(1)-\mathrm{C}(18)$ & $-34.51(11)$ \\
$\mathrm{O}(3)-\mathrm{Pd}(1)-\mathrm{P}(1)-\mathrm{C}(18)$ & $5.0(3)$ \\
$\mathrm{C}(3)-\mathrm{Pd}(1)-\mathrm{P}(1)-\mathrm{C}(6)$ & $141.72(9)$ \\
$\mathrm{O}(2)-\mathrm{Pd}(1)-\mathrm{P}(1)-\mathrm{C}(6)$ & $-119.71(18)$ \\
$\mathrm{O}(3)-\mathrm{Pd}(1)-\mathrm{P}(1)-\mathrm{C}(6)$ & $-150.72(10)$ \\
$\mathrm{C}(12)-\mathrm{P}(1)-\mathrm{C}(6)-\mathrm{C}(11)$ & $-111.2(3)$ \\
$\mathrm{C}(18)-\mathrm{P}(1)-\mathrm{C}(6)-\mathrm{C}(11)$ & $25.51(9)$ \\
$\mathrm{Pd}(1)-\mathrm{P}(1)-\mathrm{C}(6)-\mathrm{C}(11)$ & $-90.6(2)$ \\
$\mathrm{C}(12)-\mathrm{P}(1)-\mathrm{C}(6)-\mathrm{C}(7)$ & $18.7(2)$ \\
$\mathrm{C}(18)-\mathrm{P}(1)-\mathrm{C}(6)-\mathrm{C}(7)$ & $140.12(17)$ \\
$\mathrm{Pd}(1)-\mathrm{P}(1)-\mathrm{C}(6)-\mathrm{C}(7)$ & $88.16(19)$ \\
$\mathrm{C}(11)-\mathrm{C}(6)-\mathrm{C}(7)-\mathrm{C}(8)$ & $-162.60(18)$ \\
$\mathrm{P}(1)-\mathrm{C}(6)-\mathrm{C}(7)-\mathrm{C}(8)$ & $-41.14(19)$ \\
$\mathrm{C}(11)-\mathrm{C}(6)-\mathrm{C}(7)-\mathrm{S}(1)$ & $-0.9(3)$ \\
$\mathrm{P}(1)-\mathrm{C}(6)-\mathrm{C}(7)-\mathrm{S}(1)$ & $-179.68(17)$ \\
$\mathrm{C}(8)-\mathrm{C}(7)-\mathrm{S}(1)-\mathrm{O}(5)$ & $-17)$ \\
$\mathrm{C}(6)-\mathrm{C}(7)-\mathrm{S}(1)-\mathrm{O}(5)$ & \\
$\mathrm{C}(8)-\mathrm{C}(7)-\mathrm{S}(1)-\mathrm{O}(4)$ & \\
$\mathrm{C}(6)-\mathrm{C}(7)-\mathrm{S}(1)-\mathrm{O}(4)$ & \\
$\mathrm{C}(8)-\mathrm{C}(7)-\mathrm{S}(1)-\mathrm{O}(3)$ & \\
$\mathrm{C}(6)-\mathrm{C}(7)-\mathrm{S}(1)-\mathrm{O}(3)$ & \\
& \\
& \\
&
\end{tabular}




$\begin{array}{lc}\mathrm{O}(5)-\mathrm{S}(1)-\mathrm{O}(3)-\mathrm{Pd}(1) & 174.05(10) \\ \mathrm{O}(4)-\mathrm{S}(1)-\mathrm{O}(3)-\mathrm{Pd}(1) & 43.53(13) \\ \mathrm{C}(7)-\mathrm{S}(1)-\mathrm{O}(3)-\mathrm{Pd}(1) & -71.13(12) \\ \mathrm{C}(3)-\mathrm{Pd}(1)-\mathrm{O}(3)-\mathrm{S}(1) & -177.8(5) \\ \mathrm{O}(2)-\mathrm{Pd}(1)-\mathrm{O}(3)-\mathrm{S}(1) & -156.37(11) \\ \mathrm{P}(1)-\mathrm{Pd}(1)-\mathrm{O}(3)-\mathrm{S}(1) & 29.23(10) \\ \mathrm{C}(6)-\mathrm{C}(7)-\mathrm{C}(8)-\mathrm{C}(9) & 0.3(3) \\ \mathrm{S}(1)-\mathrm{C}(7)-\mathrm{C}(8)-\mathrm{C}(9) & -179.00(18) \\ \mathrm{C}(7)-\mathrm{C}(8)-\mathrm{C}(9)-\mathrm{C}(10) & 1.0(3) \\ \mathrm{C}(8)-\mathrm{C}(9)-\mathrm{C}(10)-\mathrm{C}(11) & -1.7(3) \\ \mathrm{C}(9)-\mathrm{C}(10)-\mathrm{C}(11)-\mathrm{C}(6) & 1.1(4) \\ \mathrm{C}(7)-\mathrm{C}(6)-\mathrm{C}(11)-\mathrm{C}(10) & 0.2(3) \\ \mathrm{P}(1)-\mathrm{C}(6)-\mathrm{C}(11)-\mathrm{C}(10) & 179.02(17) \\ \mathrm{C}(18)-\mathrm{P}(1)-\mathrm{C}(12)-\mathrm{C}(13) & 124.57(19) \\ \mathrm{C}(6)-\mathrm{P}(1)-\mathrm{C}(12)-\mathrm{C}(13) & -126.12(19) \\ \mathrm{Pd}(1)-\mathrm{P}(1)-\mathrm{C}(12)-\mathrm{C}(13) & -2.7(2) \\ \mathrm{C}(18)-\mathrm{P}(1)-\mathrm{C}(12)-\mathrm{C}(17) & -56.2(2) \\ \mathrm{C}(6)-\mathrm{P}(1)-\mathrm{C}(12)-\mathrm{C}(17) & 53.1(2) \\ \mathrm{Pd}(1)-\mathrm{P}(1)-\mathrm{C}(12)-\mathrm{C}(17) & 176.49(15) \\ \mathrm{C}(17)-\mathrm{C}(12)-\mathrm{C}(13)-\mathrm{C}(14) & 0.8(3) \\ \mathrm{P}(1)-\mathrm{C}(12)-\mathrm{C}(13)-\mathrm{C}(14) & -179.98(18) \\ \mathrm{C}(12)-\mathrm{C}(13)-\mathrm{C}(14)-\mathrm{C}(15) & -0.6(4) \\ \mathrm{C}(13)-\mathrm{C}(14)-\mathrm{C}(15)-\mathrm{C}(16) & 0.0(4) \\ \mathrm{C}(14)-\mathrm{C}(15)-\mathrm{C}(16)-\mathrm{C}(17) & 0.4(4) \\ \mathrm{C}(15)-\mathrm{C}(16)-\mathrm{C}(17)-\mathrm{C}(12) & -0.1(3) \\ \mathrm{C}(13)-\mathrm{C}(12)-\mathrm{C}(17)-\mathrm{C}(16) & -0.5(3) \\ \mathrm{P}(1)-\mathrm{C}(12)-\mathrm{C}(17)-\mathrm{C}(16) & -176.9(2) \\ \mathrm{C}(12)-\mathrm{P}(1)-\mathrm{C}(18)-\mathrm{C}(19) & -179.67(18) \\ \mathrm{C}(6)-\mathrm{P}(1)-\mathrm{C}(18)-\mathrm{C}(19) & 166.00(18) \\ \mathrm{Pd}(1)-\mathrm{P}(1)-\mathrm{C}(18)-\mathrm{C}(19) & 56.7(2) \\ \mathrm{C}(12)-\mathrm{P}(1)-\mathrm{C}(18)-\mathrm{C}(23) & -62.8(2) \\ \mathrm{C}(6)-\mathrm{P}(1)-\mathrm{C}(18)-\mathrm{C}(23) & -18.4(2) \\ \mathrm{Pd}(1)-\mathrm{P}(1)-\mathrm{C}(18)-\mathrm{C}(23) & -127.7(2) \\ \mathrm{C}(23)-\mathrm{C}(18)-\mathrm{C}(19)-\mathrm{C}(20) & 112.77(19) \\ \mathrm{P}(1)-\mathrm{C}(18)-\mathrm{C}(19)-\mathrm{C}(20) & 1.8(4) \\ \mathrm{C}(18)-\mathrm{C}(19)-\mathrm{C}(20)-\mathrm{C}(21) & \\ \mathrm{C}(19)-\mathrm{C}(20)-\mathrm{C}(21)-\mathrm{C}(22) & 0.35) \\ \mathrm{C}(20)-\mathrm{C}(21)-\mathrm{C}(22)-\mathrm{C}(23) & \\ \mathrm{C}(21)-\mathrm{C}(22)-\mathrm{C}(23)-\mathrm{C}(18) & \\ \mathrm{C}(19)-\mathrm{C}(18)-\mathrm{C}(23)-\mathrm{C}(22) & \\ \mathrm{P}(1)-\mathrm{C}(18)-\mathrm{C}(23)-\mathrm{C}(22) & \\ & \end{array}$

